UNDERGRADUATE RESEARCH IN NATURAL AND CLINICAL SCIENCE AND TECHNOLOGY (URNCST) JOURNAL Read more URNCST Journal articles and submit your own today at: https://www.urncst.com

\title{
The 5th Annual CCNM Research Day: Student Research \& Innovation in Naturopathic Medicine
}

Valentina Cardozo, MSc [1], Monique Aucoin, ND [1], Mohamed Elsayed, ND [1], Neda Ebrahimi, PhD [1], Anna Garber, MSc [1], Kieran Cooley, ND [1-4]*

[1] Canadian College of Naturopathic Medicine, Toronto, Ontario, Canada M2K 1E2

[2] University of Technology Sydney, Ultimo, Australia

[3] Pacific College of Health Sciences, San Diego, USA

[4] National Centre for Naturopathic Medicine, Southern Cross University, Lismore, Australia

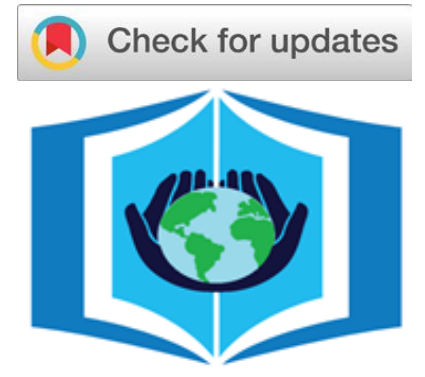

URNCST Journal

"Research in Earnest"

*Corresponding Author: kcooley@ccnm.edu

\begin{abstract}
The following are abstracts from the research competition at the 5th annual CCNM Research Day hosted by the Canadian College of Naturopathic Medicine in Toronto, ON, Canada. The conference celebrates high quality student-faculty research collaborations, showcased as poster presentations.
\end{abstract}

Keywords: naturopathy; research; naturopathic medicine; complementary medicine; integrative medicine; undergraduate research competition; innovation; mentors

Table of Contents

Poster Presentation Abstracts.

pg. A01-A11

Note: Research posters associated with this conference abstract book are available as a supplementary file.

Conference Abstracts

Note: These abstracts have been reproduced directly from the material supplied by the authors, without editorial alteration by the staff of the URNCST Journal. Insufficiencies of preparation, grammar, spelling, style, syntax, and usage are the authors.

\section{Poster Presentation Abstracts}

Dietary counseling in schizophrenia spectrum disorders: Creation and evaluation of an evidence-informed psychoeducational tool Monique Aucoin, ND [1], Laura LaChance, MD [2], Kieran Cooley, ND [1, 3-5]

[1] Canadian College of Naturopathic Medicine, Toronto, Ontario, Canada

[2] McGill University, Montreal, Quebec, Canada

[3] University of Technology Sydney, Ultimo, Australia

[4] Pacific College of Health Sciences, San Diego, USA

[5] National Centre for Naturopathic Medicine, Southern Cross University, Lismore, Australia

Background: Schizophrenia spectrum disorders (SSD) are a group of severe, persistent mental illnesses. Evidence is mounting to support the role of nutrition in mental illness; however, nutrition counselling is infrequently provided to this population. A recent scoping review collated the research on the association between diet and psychosis symptoms.

Objective: The objective of this knowledge translation project was to create an evidence-informed tool and clinician guide to facilitate discussion about nutrition between clinicians and patients with SSD.

Methods: It included an evaluation phase with a focus group involving five psychiatrists and individual phone interviews with six individuals with lived experience to obtain feedback on the initial draft. Interviews included multiple choice and open-ended questions. Interviews were recorded, transcribed and data was sorted into the following categories: 1) Content/Information, 2) Complexity, 3) Structure/Orientation, 4) Layout/Design, 5) Patient Centeredness, and 6) Nutrition as 
UNDERGRADUATE RESEARCH IN NATURAL AND CLINICAL SCIENCE AND TECHNOLOGY (URNCST) JOURNAL Read more URNCST Journal articles and submit your own today at: https://www.urncst.com

a blind spot in psychiatric care. Interpretation of the data and revisions occurred through discussion and consensus. A brief phone call will be completed with all psychiatrists and individuals to obtain feedback on the revised draft.

Results: All individuals with lived experience of mental illness rated the tool as extremely informative, easy to understand and useful. Psychiatrists primarily rated the tool and clinicians guide as extremely interesting and informative, somewhat easy to understand and somewhat useful. Responses to the open-ended questions included positive comments about the diet recommendations, level of complexity, level of participant interaction, design, and feasibility. Constructive comments included suggestions to add more sample meals, simplify recommendations, improve motivational enhancement, clearly identify action items, and increase sensitivity to culture and financial barriers. A lack of training, knowledge and use of nutritional counselling in psychiatric practice was a common theme cited by both groups of participants. The feedback was used to create a revised version of the tool. Feedback on the clinician guide was used to improve the presentation of supporting research.

Discussion and Conclusions: This tool and clinician guideline may help to facilitate a discussion about diet and mental health between patients and clinicians. The evaluation process increases the likelihood that the final tool will meet the needs of the target population.

Funding Sources: This research was funded by a Canadian CAM Research Fund grant.

Conflict of Interest: The authors declare no conflict of interest.

\section{Diet and anxiety: A scoping review}

Monique Aucoin, ND [1], Laura LaChance, MD [2], Daniella Remy, CCNM Student [1,3], Tanisha Shekdar, CCNM Student [1], Negin Sayar, CCNM Student [1], Tara Rawana CCNM Student [1], Irina Chan, CCNM Student [1], Valentina Cardozo, MSc [1], Neda Ebrahimi, PhD [1], Kieran Cooley, ND [1, 4-6]

[1] Canadian College of Naturopathic Medicine, Toronto, Ontario, Canada

[2] McGill University, Montreal, Quebec, Canada

[3]Anthrophi Technologies Ltd (ph360.me), Australia

[4] University of Technology Sydney, Ultimo, Australia

[5] Pacific College of Health Sciences, San Diego, USA

[6] National Centre for Naturopathic Medicine, Southern Cross University, Lismore, Australia

Background: Anxiety disorders are the most common group of mental disorders. They lead to significant disability and are often inadequately treated. There is mounting evidence demonstrating the important role of nutrition in the development and progression of mental disorders such as depression; however, less is known about the role of nutrition in anxiety disorders. This scoping review sought to systematically map out the body of existing literature on anxiety disorders and nutrition in order to identify effective nutritional interventions for the prevention or treatment of anxiety symptoms as well as identify gaps and opportunities for further research.

Methods: The review followed established methodological approaches for scoping reviews including an extensive $a$ priori search strategy of two databases (MEDLINE and EMBASE), duplicate screening, and standardized data extraction. Due to the large volume of results, an online program (Abstrackr) was used for concurrent screening and tagging by dietary constituent. Studies reporting an association with increased or decreased anxiety symptoms were counted and presented in figures.

Results: 55914 unique results were identified by the search. After title and abstract screening, 2213 articles were included. After interim full-text analysis, 1345 articles have been included. Preliminary data analysis revealed a possible protective effect of fruits and vegetables, omega-3 fatty acids, healthy dietary patterns, caloric restriction, breakfast consumption, ketogenic diet, broad-spectrum micronutrient supplementation, zinc, and selenium. Possibly harmful dietary constituents include: High-fat diet, inadequate tryptophan and dietary protein, high intake of sugar and refined carbohydrates, and unhealthy dietary patterns.

Discussion and Conclusions: Findings are consistent with established evidence about healthy eating patterns and mental health. Our review includes a large percent of animal and observational studies which limits the strength of our conclusions. Only a small number of intervention studies involved clinical populations of participants with anxiety disorders, many involved healthy participants or individuals with other illnesses limiting the applicability of the finding. High quality intervention studies involving participants with anxiety disorders are warranted.

Funding Sources: No funding was received for the conduct of this research

Conflict of Interest: The authors declare no conflict of interest

Cardozo et al. | URNCST Journal (2021): Volume 5, Issue 3

Page A2 of A12

DOI Link: https://doi.org/10.26685/urncst.253 
UNDERGRADUATE RESEARCH IN NATURAL AND CLINICAL SCIENCE AND TECHNOLOGY (URNCST) JOURNAL Read more URNCST Journal articles and submit your own today at: https://www.urncst.com

Naturopathic therapies as adjunctive treatment for chemotherapy-induced nausea and vomiting: A narrative review Brittany Sampson, BScHNU (Hon), CCNM student [1], Daniel Lander, ND [1]

[1] Canadian College of Naturopathic Medicine, Toronto, Ontario, Canada

Background: Chemotherapy-induced nausea and vomiting (CINV) is one of the most common and debilitating adverse effects experienced by people living with cancer, diminishing their quality of life or most concerning, leading to the discontinuation of life-saving therapies. Current guidelines recommend a prophylactic polypharmacy approach. Despite these protocols, 50-60\% of patients continue to experience CINV. The objective of this study was to investigate the efficacy of acupuncture, Zingiber officinale and Viscum album as adjunctive therapies to standard pharmaceutical antiemetics in the treatment of CINV.

Methods: PubMed, Cochrane and KNOW databases were searched using the terms chemo, chemotherapy, ginger, Zingiber officinale, mistletoe, Viscum album, and acupuncture. Studies were included if they were randomized control trials, systematic reviews or meta-analysis of adults with any type of cancer receiving chemotherapy and pharmaceutical antiemetics. Non-English language articles were excluded. The articles were assessed for change in nausea and vomiting frequency, severity or incidence and a narrative synthesis of the results was presented.

Results: Nine studies were included. Acupuncture was reported in a systematic review to be more effective than antiemetics alone at reducing the frequency and intensity of nausea and reducing the need for rescue antiemetics. In an RCT, acupuncture was reported to have better control over delayed CINV and equivalent control of acute CINV when compared to IV ondansetron. Ginger studies reported inconsistent results. One RCT found ginger to be no different than placebo. A metaanalysis favored ginger (OR 0.60) for the management of acute CINV. Viscum album had conflicting evidence. There was a trend in favor of Viscum album in the management of CINV in two independent studies.

Discussion: The majority of the studies included had small sample sizes, high heterogeneity and a high risk of bias. Additional high-quality studies are needed to continue to investigate the clinical effectiveness of mistletoe. Ginger may be effective in the management of acute CINV. More studies are needed to determine the most effective dose. There is some evidence to support the use of acupuncture as a part of a comprehensive treatment plan for CINV.

Funding Sources: No funding was received for the conduct of this research

Conflict of Interest: The authors declare no conflict of interest

Investigating non-pharmacological interventions for the treatment of fibromyalgia related pain: A narrative review Paige Weatherbee, CCNM Student [1], Louise McCrindle, ND [1]

[1] Canadian College of Naturopathic Medicine, Toronto, Ontario, Canada

Background: Fibromyalgia is a chronic pain syndrome that affects approximately $6 \%$ of Canadians. The primary component of the diagnostic criteria is musculoskeletal pain, but other symptoms include fatigue, nonrestorative sleep, cognitive dysfunction and mood disorders. Current standards of care encourage the use of non-pharmacological interventions and the Canadian guidelines point to acupuncture, Transcutaneous Electrical Nerve Stimulation (TENS), and Cognitive Behavioural Therapy (CBT) as possible treatment options. Discerning the effectiveness of these non-pharmacological interventions may allow for better treatment of chronic pain related to fibromyalgia and therefore an improvement in quality of life. The purpose of this review is to discuss the effectiveness of these interventions based on the available literature.

Methods: The PubMed and Medline databases were searched using different combinations of the terms fibromyalgia, acupuncture, TENS, Transcutaneous Electrical Nerve Stimulation, CBT, and Cognitive Behavioral Therapy. The literature search was restricted to full text articles, Meta-Analysis, randomized controlled trials (RCT), and only included studies that reported changes in chronic pain. Meta-analyses were excluded if there was more than 50\% overlap of included studies (only the most recent or the largest were included), used combination therapy, were not in English, or if they did not report findings using a visual analog or comparable point scale.

Results: 6 Meta-analyses and 4 RCTs were included for analysis. Evidence for acupuncture showed a trend toward effectiveness in pain reduction post-treatment, but there was no consensus as to the effectiveness post treatment. Evidence for TENS was promising if the device was used while active but was not seen to be effective if worn solely while at rest. CBT evidence was statistically significant for pain reduction across the board regardless of in person, over the phone, or webbased delivery. Additionally, both TENS and CBT showed promise for pain reduction when employed by subjects at home to allow for pacing. The strength of evidence however remained low for all three interventions.

Cardozo et al. | URNCST Journal (2021): Volume 5, Issue 3

Page A3 of A12

DOI Link: https://doi.org/10.26685/urncst.253 
UNDERGRADUATE RESEARCH IN NATURAL AND CLINICAL SCIENCE AND TECHNOLOGY (URNCST) JOURNAL Read more URNCST Journal articles and submit your own today at: https://www.urncst.com

Conclusions: Acupuncture, TENS, and CBT are all viable options for reduction of chronic pain with Fibromyalgia; however, more research with larger trials, longer follow-up, and improved quality are required in order to make definitive statements on the effectiveness of these non-pharmacological treatments. Additionally, more specific research on TENS and CBT for at home treatment in order to overcome accessibility fatigue and financial related barriers is warranted.

Funding Sources: No funding was received for the conduct of this research

Conflict of Interest: The authors declare no conflict of interest

Vitamin $\mathbf{C}$ for treating depression in cancer patients: A literature review

Jordan Kerner, CCNM Student [1], Erica Eckstrand, CCNM Student [1], Monique Aucoin, ND [1]

[1] Canadian College of Naturopathic Medicine, Toronto, Ontario, Canada

Background: One in two Canadians will get cancer in their lifetime and one in four will experience depression during their diagnosis. The cause of depression during cancer could be related to psychological, social, inflammatory or immunological factors. Vitamin $\mathrm{C}$ can exert an effect on oxidative stress levels, adrenal function and immune function, and may have a role in the treatment of both cancer and depression. Previous research has shown that vitamin $\mathrm{C}$ can affect neurotransmitter levels, improve quality of life, and relieve other cancer-related symptoms such as pain. This literature review was conducted in order to identify all studies that assessed changes in depression symptom severity in cancer patients receiving intravenous vitamin C treatment.

Methods: PubMed, MedLine Complete, CINAHL Plus, Web of Science, Cochrane, and PMC databases were searched in May 2020. Search terms included "IVC", "Intravenous Vitamin C", "Vitamin C", and "Ascorbic Acid" in conjunction with the terms "Depression" and "Cancer." Bibliographies of relevant articles were searched for additional publications. We included both prospective and retrospective observational studies. The inclusion criteria consisted of human participants with confirmed cancer (any type and stage), intravenous vitamin $\mathrm{C}$ treatment (IVC), with or without conventional treatment and with or without additional oral vitamin $\mathrm{C}$ dosing, and an assessment of depression. Preclinical studies (animal, in vitro), nonEnglish publications, literature reviews and opinion articles were excluded.

Results: This review identified 152 unique articles and four observational studies met the inclusion criteria. All four studies that were identified reported improvement in mood.

Discussion: The studies included in this review align with previous research in reporting that IVC improved quality of life and had other cancer-related benefits (i.e., decreasing fatigue and pain). Furthermore, the studies all evaluated the safety of IVC and reported no significant adverse effects associated with IVC. These findings are limited by a lack of control group and the lack of use of validated questionnaires to measure depression symptoms.

Conclusion: The results presented suggest that IVC could potentially have a beneficial effect on levels of depression in patients with cancer; however, more research is needed.

Funding Sources: No funding was received for the conduct of this research

Conflict of Interest: The authors declare no conflict of interest

Evidence for Arnica montana's use in physical trauma: A narrative review

Brittany Harris, DCHM, CCNM Student [1], Paul Richard Saunders, PhD, ND [1-3]

[1] Canadian College of Naturopathic Medicine, Toronto, Ontario, Canada

[2] Canadian College of Homeopathic Medicine, Toronto, Ontario, Canada

[3] National University of Health Science, Chicago, USA

Introduction: Arnica montana (Asteraceae) has been used to ameliorate the symptoms associated with physical trauma for over 400 years. Information on this plant is found in a multitude of different sources; botanical materia medicas, homoeopathic materia medicas, and modern databases of clinical research. Practitioners therefore do not always know of, or know how to find, all of the available evidence. For example, proving data (homœopathic evidence on an intervention's usage) is not available in online medical databases like PubMed. Similarly old (200+ years) case studies are not usually indexed on databases like PubMed, but are aggregated in botanical materia medicas. The purpose of this review is to aggregate the different types of evidence to form a complete understanding of the evidence for Arnica's use in trauma.

Methods: A search of PubMed, five homœopathic materia medicas and five botanical materia medicas was conducted. The homœopathic sources investigated were Hahnemann's Materia Medica Pura, Vithoulkas' Materia Medica Viva, Hering's Guiding Symptoms, Allen's Encyclopedia and Lippe's Textbook of Materia Medica. For botanical medicine Scudder's Specific Medication and Specific Medicines, King's American Dispensary by Felter and Lloyd, Woodville's Medical Botany, Hoffmann's Medical Herbalism and Godfrey and Saunders' Naturopathic Botanical Medicine were consulted. Clinical trials

Cardozo et al. | URNCST Journal (2021): Volume 5, Issue 3

DOI Link: https://doi.org/10.26685/urncst.253

Page A4 of A12 
UNDERGRADUATE RESEARCH IN NATURAL AND CLINICAL SCIENCE AND TECHNOLOGY (URNCST) JOURNAL Read more URNCST Journal articles and submit your own today at: https://www.urncst.com

and materia medicas can contain very different types of data. In order to be able to compare them, the endpoints listed in the trials were used as search terms to find the same information in the materia medicas. Any source that studied Arnica mixed with other substances was excluded. An abstract or full text in English had to be available for sources to be included.

Results: 37 studies found on PubMed were included. Of these, the most common endpoint was postoperative healing. Healing was tracked by pain, ecchymosis and edema most commonly. The most common surgery studied was rhinoplasty. Studies only included a few endpoints per study. In comparison, every homoeopathic materia medica included information on every single endpoint except for infection (no materia medica contained information on general infections). The botanical materia medicas had many of the endpoints, but not all.

Discussion: There is an incredible amount of evidence from biomedical and traditional sources evaluating Arnica's use in trauma. However, the lens through which you look at the evidence determines how you interpret the information and how its utilized in practice. Case reports and articles on clinical experience, which make up the bulk of materia medicas, are nuanced and include a large variety of indications and differentiating factors (i.e. when to use Arnica vs another intervention in physical trauma). Clinical trials are incredibly specific; clinical trials investigate whether an intervention changes a specific endpoint in a specific population, at a specific dose. Because materia medicas and clinical trials contain different kinds of data, it would behoove practitioners to be familiar with the content, merits, and deficiencies of each.

Funding Sources: No funding was received for the conduct of this research

Conflict of Interest: The authors declare no conflict of interest

\author{
A scoping review of botanical medicine therapies for first-line clinical treatment of polycystic ovarian syndrome \\ Amy Storozuk, H.BSc, CCNM Student [1], Anna Garber, M.Sc, CCNM Student [1], Colton Schwenk, ND [1], \\ Teresa Tsui, ND [1] \\ [1] Canadian College of Naturopathic Medicine, Toronto, Ontario, Canada
}

Background: Polycystic Ovarian Syndrome (PCOS) is a complex endocrine disorder affecting 5-10\% of reproductive aged women. The Endocrine Society recommends that PCOS be diagnosed using the Rotterdam criteria, which requires the presence of two of three symptoms - oligomenorrhea, hyperandrogenism, and polycystic ovaries on ultrasound - and the exclusion of other diagnoses of hyperandrogenism or ovulatory dysfunction. First-line management of PCOS often involves pharmaceuticals and lifestyle modification. Pharmaceutical treatment alone addresses single symptoms, may be contraindicated, is often associated with side effects, and may not always be effective, hence emphasis is placed on a multidisciplinary approach.

Botanical medicines, involving complex mechanisms of action, have been used safely for hundreds of years to manage PCOS. This scoping review updates the Arentz et al. 2014 scoping review with a focus on human female subjects to inform best clinical practices for the first-line management of PCOS with botanical medicine.

Methods: Three PubMed searches were conducted for: 1) complementary therapies for PCOS/ hyperandrogenism; 2) a hand search of relevant articles from the references of our initial search; and 3) inclusion of additional synonyms for "botanical medicine" due to limited initial search results. Inclusion criteria was: human female subjects, diagnosis of PCOS, use of herbal medicine as first-line treatment for PCOS, herbal medicines with a demonstrated mechanism of reproductive endocrinological effect, and clinical studies investigating commercially available herbal extracts.

Results: Twenty studies were included in this review. All studies reported mechanisms of action, including 3 reviews with a total of 99 studies and 5858 women with PCOS and/or hyperandrogenism. Botanical interventions included Cimicifuga racemosa, Cinnamomum cassia, Glycyrrhiza glabra, Paeonia lactiflora, Mentha spicata, Tribulus terrestris, Unkei-to, and Vitex agnus-castus. Endocrine outcomes included lowered prolactin, luteinizing hormone, androgens, and ovarian volume; increased estradiol and follicle stimulating hormone; menstrual regularity; and improved insulin sensitivity. Other outcomes were improved mood and cognition, lipid profile, and quality of life.

Conclusions: This scoping review summarizes ten botanicals, six botanical combinations, three traditional Chinese medicinal herbal formulas, and three natural health products as evidence-based options for treating PCOS and hyperandrogenism.

Funding Sources: No funding was received for the conduct of this research

Conflict of Interest: The authors declare no conflict of interest

Cardozo et al. | URNCST Journal (2021): Volume 5, Issue 3

Page A5 of A12

DOI Link: https://doi.org/10.26685/urncst.253 
UNDERGRADUATE RESEARCH IN NATURAL AND CLINICAL SCIENCE AND TECHNOLOGY (URNCST) JOURNAL Read more URNCST Journal articles and submit your own today at: https://www.urncst.com

\author{
Natural interventions for improving quality of life in those with multiple sclerosis: A narrative review \\ Samantha Murray, CCNM Student [1], Adam Gratton, ND [1] \\ [1] Canadian College of Naturopathic Medicine, Toronto, Ontario, Canada
}

Background: Approximately, 77,000 people in Canada are diagnosed with multiple sclerosis (MS). Although there has been progress with treatment of this disease there is no cure and MS can be a debilitating disease affecting people across every aspect of their life. There is a high rate of heterogeneity in the presentation of symptoms for those with MS as such a focus on overall quality of living can be enlightening. Many people with MS turn to non-pharmacological interventions to help address quality of life or symptom management despite an unclear evidence base. The evidence for safety and effectiveness of 3 natural interventions will be summarized in this review: progressive resistance training, mindfulness-based stress reduction (MBSR) and vitamin D supplementation.

Methods: Using PubMed, Google Scholar and Cochrane Library, three searches were conducted. Parameters were set to 1) adults with multiple sclerosis, 2) comparison of the intervention group to a control group using standard care or to baseline measures, and 3) outcomes assessments for quality of life. Limits were used to refine results to publication dates in 2010 2020, randomized control trials, systematic reviews, and meta-analyses, humans, and English to ensure academic integrity of the information collected.

Results: A total of 4 articles on progressive resistance training, 3 on MBSR, and 3 on Vitamin D were selected for this review, other articles were available on the topics but were either covered in meta-analyses already included or did not report sufficient information on quality of life to be included in the review.

Conclusion: The evidence suggests that all three interventions show promise for improving quality of life in those with MS. Inconsistencies in how the intervention was delivered amongst the studies suggests that set protocols or best practices have yet to be established. These findings are promising and call for further research, and provide some considerations for implementing in clinical practice according to patient ability and willingness.

Funding Sources: No funding was received for the conduct of this research

Conflict of Interest: The authors declare no conflict of interest

Effectiveness of naturopathic care for anxiety and depression: A retrospective chart review of a teaching clinic Joy Dertinger ND [1], Simone Singh MD, DipIBLM, CCNM Student [1], Shaida Farrokhi, CCNM Student [1], Amalia Stephenson, CCNM Student [1], Zachary De Rose, CCNM Student [1]

[1] Canadian College of Naturopathic Medicine, Toronto, Ontario, Canada

Background: About $10 \%$ of Canadians seek services for mood and anxiety disorders annually. Some patients may be more likely to seek Naturopathic services due to stigma.Evidence for individual therapies and a few whole-systems studies support the use of Naturopathic Medicine for anxiety and depression. This study aims to describe the characteristics of patients with anxiety and depression on a focus shift at a Naturopathic teaching clinic and evaluate the effectiveness of care.

Methods: Electronic medical charts from the Mental Health Shift (MHS) at the Robert Schad Naturopathic Clinic (RSNC) were reviewed for demographics, treatments, compliance, and response to treatment. Charts were selected by reviewing every patient to visit the MHS between January 1, 2019 and March 6, 2020 and selecting patients with anxiety and/or depression in their charted assessment. Subjects were included if their initial GAD7 or PHQ9 score was at least 10 and scores were at least four weeks apart. Change in initial and final GAD-7 and PHQ-9 scores was evaluated using two-tailed paired T-tests. This study was approved by the Canadian College of Medicine's Research and Ethics Board.

Participants: Of the 192 charts reviewed, 22 met the inclusion criteria. The patients were 64\% female (14/22), aged 20-68 years old (mean age 37 ) and visited the MHS an average of 1.8 times per month.

Results: All participants received recommendations for natural health products, averaging five products each. The most common were lavender, B-complex, fish oil, magnesium, melatonin and rhodiola. Most (14/22, 64\%) subjects also received a lifestyle recommendation involving diet, exercise, mindfulness, goal-setting and/or psycho-education. Mild adverse events were reported in 6 charts $(27 \%)$. Mean PHQ9 and GAD7 scores decreased 7 ( $p<0.0001)$ and 5 (p<0.001) points respectively. Subjective improvement was noted in $73 \%(16 / 22)$ of charts. A clinically significant decrease of $\geq 5$ points was seen in $73 \%$ $(16 / 22)$ of PHQ9 scores and 55\% (12/22) of GAD7 scores.

Conclusion: Naturopathic care as performed on the MHS is effective in reducing some depression and anxiety symptoms in most patients. Further research incorporating comparison groups are warranted.

Funding Sources: No funding was received for the conduct of this research

Conflict of Interest: The authors declare no conflict of interest

Cardozo et al. | URNCST Journal (2021): Volume 5, Issue 3

Page A6 of A12

DOI Link: https://doi.org/10.26685/urncst.253 
UNDERGRADUATE RESEARCH IN NATURAL AND CLINICAL SCIENCE AND TECHNOLOGY (URNCST) JOURNAL Read more URNCST Journal articles and submit your own today at: https://www.urncst.com

The effect of community involvement on anxiety and depression: A narrative review

Alessandra Perciballi CCNM Student [1] and Adam Gratton MSc ND [1]

[1] Canadian College of Naturopathic Medicine, Toronto, Ontario, Canada

Background: Mental health concerns, like anxiety and depression, are frequently expressed to primary care providers. Current standards of care involve a combination of drug and psychotherapy options. These options can be costly, inaccessible, time consuming, and with pharmaceutical options, involve the potential for adverse effects. Treatment success is not guaranteed, which fuels patient desire for adjunctive or alternative interventions. Social engagement has been investigated as a potential option to improve outcomes related to anxiety and depression. In this narrative review, social engagement within the context of community volunteerism or gardening are explored for their efficacy on various mental health outcomes such as feelings of social connectedness, subjective well-being, quality of life, and self-esteem in people with anxiety or depression.

Methods: PubMed was searched looking for articles of any methodology published between 2016-2020. Since the focus of the study explored the broad topic of all community involvement, two separate searches were conducted. The first search used the following terms: community garden AND (mental health OR depression OR anxiety). The second search used the following terms: (Volunteer OR Volunteering) AND (mental health OR depression OR anxiety). Studies included must have reported outcomes related to depression and/or anxiety using any validated outcomes measure. Studies involving professional volunteer environments, like volunteer firefighting, were excluded.

Results: The literature searches yielded a total of 54 studies of which 7 articles met the inclusion and exclusion criteria comprising 4 qualitative studies, 1 systematic review, 1 randomized controlled trial, and 1 observational cohort study. The results of these studies indicate almost exclusively positive benefit of community involvement on mental health outcomes whether that be self-directed, other-directed, or community gardening. Community involvement was able to improve mental health, depressive symptoms, quality of life, life satisfaction, self-esteem, cognitive functioning, and social connectedness. Of importance to note is that despite some trials mandating volunteer work, the positive benefit was maintained.

Conclusion: Community involvement may be beneficial at reducing symptoms of depression or anxiety. In addition to improving quality of life outcomes in patients, community involvement can benefit communities as a whole.

Funding Sources: No funding was received for the conduct of this research

Conflict of Interest: The authors declare no conflict of interest

Effects of maternal diets on offspring anxiety: A scoping review

Yousef Sadat Nejad CCNM Student [1], Monique Aucoin, ND [1]

[1] Canadian College of Naturopathic Medicine, Toronto, Ontario, Canada

Background: In recent decades, evidence has accumulated suggesting that dietary habits during pregnancy and early life may influence a child's subsequent vulnerability to physical and psychiatric illness, including anxiety disorders. Large population-based surveys have reported that up to $33 \%$ of Canadians are affected by an anxiety disorder during their lifetime. The purpose of this study is to systematically review the data on the relationship between maternal diet and the incidence or severity of anxiety disorders in offspring.

Methods: A comprehensive search strategy was designed by an experienced medical librarian to identify all studies that provided or measured a dietary constituent or dietary pattern in the perinatal period and assessed anxiety levels in offspring. MEDLINE and Embase databases were searched with no language or date restrictions. Titles and abstracts were screened in duplicate. Data extraction was completed using a piloted extraction template and data was analyzed qualitatively to identify trends, gaps and areas that warrant further research.

Results: After title and abstract screening, 135 human and animal studies were identified. Full text screening identified 101 studies that met criteria, including 99 animal studies and two observational human studies. Eleven of 14 studies showed an association between high fat diet and increased anxiety in offspring. Low protein intake caused an increase in offspring anxiety symptoms in 12 out of 16 studies. Other nutrients were associated with a protective effect. Thirteen of 18 studies providing adequate or additional vitamins or minerals reported a decrease in offspring anxiety. Three of four studies providing omega-3 fatty acids reported improvement. The two human observational studies reported increased anxiety with high prenatal caffeine intake and early life protein-energy malnutrition, respectively.

Cardozo et al. | URNCST Journal (2021): Volume 5, Issue 3

Page A7 of A12

DOI Link: https://doi.org/10.26685/urncst.253 
UNDERGRADUATE RESEARCH IN NATURAL AND CLINICAL SCIENCE AND TECHNOLOGY (URNCST) JOURNAL Read more URNCST Journal articles and submit your own today at: https://www.urncst.com

Discussion/Conclusion: The results of the identified studies suggest that maternal diet factors may impact the incidence of anxiety disorders in offspring. The results may be relevant in guiding recommended dietary habits for pregnant women to decrease the risk of anxiety disorder in offspring. However, the current evidence is limited by primarily animal data. More research in humans is needed.

Funding Sources: No funding was received for the conduct of this research

Conflict of Interest: The authors declare no conflict of interest

The effect of natural therapies in combination with usual care for major depressive disorder: A narrative review Prabhjot Chohan CCNM Student [1], Adam Gratton, MSc ND [1]

[1] Canadian College of Naturopathic Medicine, Toronto, Ontario, Canada

Background: Major depressive disorder (MDD) is the most common and debilitating form of depression with a 12-month prevalence of $4.7 \%$ and a lifetime prevalence of $11.2 \%$ in Canada. The various classes of antidepressants are commonly used treatments for MDD; however, high failure rates occur due to adverse events and discontinuation of use. Non-drug and alternative interventions are commonly sought by people when drug treatment fails. The purpose of this investigation was to analyze the evidence on the effect of natural therapies in combination with usual care as a treatment for MDD.

Methods: The following inclusion criteria were defined before conducting the literature search: 1) population of adults with major depressive disorder, 2) intervention of lavender, folic acid or acupuncture, combined with standard treatment, 3) comparison group of a placebo, standard treatment or natural therapy used alone, 4) changes to Hamilton Depression Rating Scale (HAM-D) as the primary outcome. PubMed, APA PsycARTICLES and Google scholar were used for the research. The articles were limited to randomized clinical trials (RCTs), and systematic reviews with meta-analyses.

Results: The literature search for lavender yielded 214 studies, of which 3 RCTs met the criteria. Folic acid yielded 680 studies of which 2 RCTs and 1 systematic review with meta-analysis met the criteria. Acupuncture yielded 2240 studies of which 2 RCTs and 2 systematic reviews with meta-analyses met the criteria. Only the RCTs not summarized in the systematic reviews and meta-analyses were summarized in this review. All ten studies using natural interventions showed a statistically significant decrease in the mean score change versus comparison groups, however, the magnitude of the effect varied between the studies. Sample sizes were small and there was significant heterogeneity between studies.

Conclusion: Evidence suggests that natural therapies can be used adjunctively to the usual care of MDD, however, the overall research quality is low and substantial heterogeneity exists between studies. Further, better designed research is warranted.

Funding Sources: No funding was received for the conduct of this research

Conflict of Interest: The authors declare no conflict of interest

The effect of echinacea supplementation on cytokine levels: A systematic review Monique Aucoin [1], Valentina Cardozo [1], Anna Garber [1], Meagan McLaren [1], Daniella Remy [1,2], Joy Dertinger [1], Mohammed Ali Kala [1], Adam Gratton [1], Alessandra Perciballi [1], Cara Warder [1], Amali Firhoj [1], Angelica Dimita [1], Kieran Cooley [1, 3-5]

[1] Canadian College of Naturopathic Medicine, Toronto, Ontario

[2] Anthrophi Technologies Ltd (ph360.me), Australia

[3] University of Technology Sydney, Ultimo, Australia

[4] Pacific College of Health Sciences, San Diego, USA

[5] National Centre for Naturopathic Medicine, Southern Cross University, Lismore, Australia

Background: COVID-19 is the respiratory illness cause by a novel coronavirus. Cytokine storm appears to be a factor in COVID-19 mortality. Echinacea species have been used historically for immune support. A previous rapid review, on the role of Echinacea in the management of respiratory tract infections in humans, suggested that Echinacea supplementation may decrease the levels of pro-inflammatory cytokines involved in cytokine storm. The objective of the present review is to systematically identify and analyze all human, animal and in vitro studies assessing the impact of Echinacea supplementation on cytokine levels.

Methods: The following databases were searched: Medline (Ovid), AMED (Ovid), CINAHL (EBSCO), EMBASE (Ovid) without date or language restriction. Titles and abstract screening, full text screening, and data extraction were completed in duplicate using a piloted extraction template. Risk of bias assessment was completed using the following tools: Cochrane Risk of Bias 2.0 (randomized clinical trials), ROBINS-I (non-randomized trials), NIH Quality Assessment Tool (pre-post

Cardozo et al. | URNCST Journal (2021): Volume 5, Issue 3

Page A8 of A12

DOI Link: https://doi.org/10.26685/urncst.253 


\section{UNDERGRADUATE RESEARCH IN NATURAL AND CLINICAL SCIENCE AND TECHNOLOGY (URNCST) JOURNAL}

Read more URNCST Journal articles and submit your own today at: https://www.urncst.com

studies with no control group), OHAT (animal studies) and Toxrtool (in vitro studies). Qualitative analysis was used to assess for trends in cytokine level changes.

Results: The search identified 264 unique publications. After title and abstract screening, 138 studies met criteria for inclusion. After full text screening, 12 human studies, including six randomized clinical trials, 20 animal studies, and 66 in vitro studies met criteria for inclusion. Among the studies involving humans, Echinacea supplementation predominantly decreased levels of the pro-inflammatory cytokine TNF- $\alpha$ and increase levels of the anti-inflammatory cytokines IFN- $\gamma$ and IFN- $\alpha$. In animal studies, Echinacea supplementation predominantly decreased levels of pro-inflammatory cytokines TNF- $\alpha$, IL-1B and IL-6 and increased levels of the anti-inflammatory cytokine IFN- $\gamma$. Analysis of in vitro data is in progress. The risk of bias in human trials was generally high.

Discussion and Conclusion: While there is currently no research on the therapeutic effects of Echinacea in the management of cytokine storm, the present evidence related to the herb's impact on cytokine levels suggest that further research may be warranted in the form of a clinical trial involving patients with COVID-19.

Funding Sources: No funding was received for the conduct of this research

Conflict of Interest: The authors declare no conflict of interest

Jarische herxheimer reaction (Candida Die-Off Like Reaction) in gastrointestinal fungal treatment with natural supplements: A case report Arvind Tuteja, CCNM Student [1], Romi Raina, ND [1]

[1] Canadian College of Naturopathic Medicine, Toronto, Ontario, Canada

Background: Jarische Herxheimer reaction (JHR) is a transient immunological response to antibiotic treatment caused by endotoxin-like products released upon microorganism death. JHR is typically described in antibiotic treatment of spirochetal infections, and other bacterial, fungal, protozoal infections; with symptoms like fever, chills, headache, myalgias, and skin lesion aggravations.

Case Presentation: A 3-year-old girl presented with atopic dermatitis with 9 months of red, itchy, dry, cracked patches that started on the elbows and behind the knees, which then spread with scarring on the chest, back and inner thighs. Comorbidities included diarrhea, runny nose and frequent colds. Treatment with omega-3s, vitamin D3, probiotics, and dietary modifications showed some relief.

Stool culture and sensitivity tests detected Rhodotorula mucilaginosa sensitive to caprylic acid and grapefruit seed extract and no growth of lactobacillus spp. The patient commenced treatment of 2 capsules per day of NFH Inc.'s Candida SAP (Allium sativum, Berberine, Oregano, Selenium, Calcium caprylate, Magnesium caprylate, Zinc caprylate). On the third day of treatment, the patient reported increased itchiness, puffy eyes, nasal congestion and fever. Diagnosing this to be a JRH or "Candida die-off like reaction", treatment was stopped and subsequently recommenced at a lower dosage with gradual stepwise increases. No further aggravation was reported, and progressive resolution of initial symptoms was observed.

Discussion: There is a paucity of literature documenting JHR after gastrointestinal (GI) fungal infection, or after treatment with a supplement preparation. We document such an occurrence, and resolution through temporary treatment discontinuation and dose reduction; whereas a mis-diagnosis may have led to stopping an otherwise efficacious treatment, erroneously considering it inefficacious or harmful. Clinicians may consider the possibility of JHR occurring as a result of either nutraceutical or pharmaceutical treatment. Commencing anti-fungal treatment in a gradual, stepwise manner may be useful in decreasing the risk of JHR.

Conclusions: This is the first documented occurrence of JHR elicited by a combination nutraceutical product and in the treatment of Rhodotorula. We hope to stimulate more reports and research related to differences in manifestations, severity and mechanism of JHR.

Funding Sources: No funding was received for the conduct of this research

Conflict of Interest: The authors declare no conflict of interest 
UNDERGRADUATE RESEARCH IN NATURAL AND CLINICAL SCIENCE AND TECHNOLOGY (URNCST) JOURNAL Read more URNCST Journal articles and submit your own today at: https://www.urncst.com

Probiotics use in patients undergoing cancer surgery: A systematic review

Elise Cogo ND, MSc, MLIS [1], Mohamed Elsayed, ND [1], Vivian Liang, ND [1], Kieran Cooley, ND [1-4], Christilynn Guerin, BSc [1], Athanasios Psihogios, ND [5], Peter Papadogianis, MSc, ND [1]

[1] Patterson Institute for Integrative Cancer Research, Canadian College of Naturopathic Medicine, Toronto, Ontario, Canada

[2] University of Technology Sydney, Ultimo, Australia

[3] Pacific College of Health Sciences, San Diego, USA

[4] National Centre for Naturopathic Medicine, Southern Cross University, Lismore, Australia

[5] Ottawa Integrative Cancer Centre, Ottawa, Ontario, Canada

Background: Probiotics are live beneficial microorganisms and they have shown promise in preventing post-operative complications. Variations in probiotic interventions and the surgical milieu require nuanced synthesis work to inform clinical decisions.

Objective: To evaluate the evidence base for the safety and efficacy of probiotics in patients with cancer during the perioperative period.

Methods: Systematic review conducted following a priori protocol registered in PROSPERO (CRD42018086168) based on methods advised by Cochrane Handbook. Primary review outcomes were: mortality, cancer treatment response, recurrence, remission, metastasis/disease progression, and stable disease. Secondary outcomes were: adverse events, post-operative infections and antibiotic use, other post-operative complications (i.e., nausea and vomiting, diarrhea, constipation, intestinal obstruction, and ileus), bleeding, length of hospitalization, quality of life, wound healing, pain, anthropometrics, fatigue, cancer biomarkers, immune cells, and inflammatory markers levels. MEDLINE, Embase and Cochrane CENTRAL were searched (inception to September 2020) for human comparative studies in English. Study selection and data extraction were done in duplicate. Risk-of-bias was appraised for randomized controlled trials (RCTs) using Cochrane Risk-of-Bias Tool, and quality assessment of observational studies was conducted with Newcastle-Ottawa Scale.

Results: 48 included articles reporting on 45 studies, comprising 37 RCTs (in 40 reports) and 8 observational cohort studies. Many diverse multi-ingredient combinations were evaluated. Four of the interventions were used in multiple RCTs, while 21 out of $37(57 \%)$ RCTs used unique products. $31(84 \%)$ RCTs investigated oral products, and $6(16 \%)$ administered interventions by enteral nutrition tubes. Probiotics administration was used only pre-operatively in 10 (27\%) RCTs, only post-operatively in 7 (19\%) RCTs, and during both surgical periods in $20(54 \%)$ RCTs. All except 3 RCTs (92\%) dealt with digestive system cancers; bladder, laryngeal, and head and neck cancers each had 1 RCT. 20 (54\%) RCTs were conducted in Asia, 11 (30\%) in Europe, 5 (14\%) in Brazil, and 1 (3\%) in USA.

Conclusions: This large and up to date evidence synthesis provides the most comprehensive systematic review on this topic. Analyses are underway.

Funding Sources: This project was funded by the Patterson Institute for Integrative Cancer Research at CCNM

Conflict of Interest: The authors declare no conflict of interest

Naturopathic management of chronic neuropathic pain: A case study

Adrienne Waunch, BSc, CCNM Student [1], Adam Gratton, ND [1]

[1] Canadian College of Naturopathic Medicine, Toronto, Ontario, Canada

Background: Chronic pain affects many Canadians and neuropathic pain can be debilitating and frustrating for many to manage. Many modalities have potential efficacy but there is a lack of evidence for many of these interventions.

Case Presentation: A 59-year-old female presented to the clinic with a diagnosis of postherpetic neuralgia (PHN) established 14 years ago, however, there were no lesions at the time of herpes zoster reinfection. A full body MRI was performed in 2019 which ruled out structural abnormalities. Burning pain has been consistently present and intense since onset despite multiple conventional treatments, including gabapentin. A protocol involving electroacupuncture $(150 \mathrm{~Hz})$ and low-level laser therapy treatments for six weeks was implemented alongside supplementation of palmitoylethanolamide (400mg TID) and alpha lipoic acid (300mg QD), and local B12 injections (1000mcg methylcobalamin). Changes in severity were systematically measured at the beginning and end of each visit using a visual analogue scale. Pre-treatment pain intensity ratings were 10/10 and post-treatment pain was reduced to 3/10. Each event of pain relief only lasted 3-6 hours post treatment, but is significant given how ineffective other treatments have been since the diagnosis.

Discussion: This combination of interventions proved effective in temporarily reducing the severity of neuropathic pain where other interventions have failed to provide any relief. Interventions with different potential mechanisms of action may prove more beneficial than relying on any one alone.

Cardozo et al. | URNCST Journal (2021): Volume 5, Issue 3

DOI Link: https://doi.org/10.26685/urncst.253

Page A10 of A12 
UNDERGRADUATE RESEARCH IN NATURAL AND CLINICAL SCIENCE AND TECHNOLOGY (URNCST) JOURNAL

Read more URNCST Journal articles and submit your own today at: https://www.urncst.com

Conclusions: This case provides more evidence for the use of naturopathic modalities in the management of chronic neuropathic pain. Although more time is needed to determine which interventions will further decrease frequency of pain for this individual, this research provides a framework for approaching future neuropathic pain cases. Further research into each of the interventions is warranted.

Funding Sources: No funding was received for the conduct of this research

Conflict of Interest: The authors declare no conflict of interest

\section{Conflicts of Interest}

The authors declare that they have no conflict of interests.

\section{Authors' Contributions}

MA and KC co-founded the first CCNM Research day.

VC, MA, ME, NE, AG and KC contributed equally to planning the research competition, assisted in the collection and review of the abstract submissions, as well as support for authors selected for the competition while producing their posters.

\section{Acknowledgements}

Special thanks to Julia Zander, Digital Media Specialist at the Canadian College of Naturopathic Medicine. Her patience, and keen, creative eye, were key to the creation of research posters for this competition. We would also like to acknowledge the work of our poster judging panel for their critical appraisal and scientific discussion on the day of the competition.

\section{Funding}

Funding for this conference has been supported by Fullscript and Biotics Research Inc. through an arms-length donation to the Canadian College of Naturopathic Medicine's Student Innovation Fund. 
UNDERGRADUATE RESEARCH IN NATURAL AND CLINICAL SCIENCE AND TECHNOLOGY (URNCST) JOURNAL Read more URNCST Journal articles and submit your own today at: https://www.urncst.com

\section{Article Information}

Managing Editor: Jeremy Y. Ng

Article Dates: Received Mar 01 21; Published Mar 0821

\section{Citation}

Please cite this article as follows:

Cardozo V, Aucoin M, Elsayed M, Ebrahimi N, Garber A, Cooley K. The 5th annual CCNM Research Day: Student research \& innovation in naturopathic medicine. URNCST Journal. 2021 Mar 08: 5(3).

https://urncst.com/index.php/urncst/article/view/253

DOI Link: https://doi.org/10.26685/urncst.253

\section{Copyright}

(C) Valentina Cardozo, Monique Aucoin, Mohamed Elsayed, Neda Ebrahimi, Anna Garber, Kieran Cooley. (2021).

Published first in the Undergraduate Research in Natural and Clinical Science and Technology (URNCST) Journal.

This is an open access article distributed under the terms of the Creative Commons Attribution License

(https://creativecommons.org/licenses/by/4.0/), which permits unrestricted use, distribution, and reproduction in any medium, provided the original work, first published in the Undergraduate Research in Natural and Clinical Science and Technology

(URNCST) Journal, is properly cited. The complete bibliographic information, a link to the original publication on http://www.urncst.com, as well as this copyright and license information must be included.
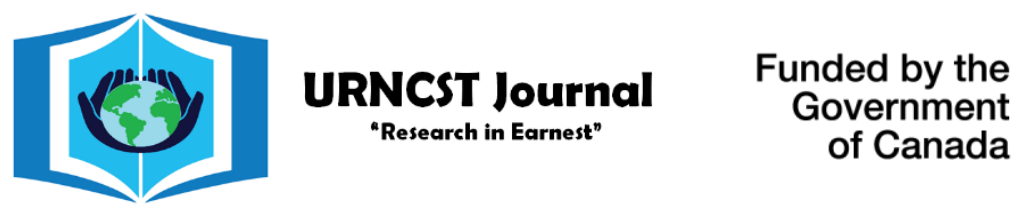

Canadà̀

Do you research in earnest? Submit your next undergraduate research article to the URNCST Journal!

| Open Access | Peer-Reviewed | Rapid Turnaround Time | International | | Broad and Multidisciplinary | Indexed | Innovative | Social Media Promoted |

Pre-submission inquiries? Send us an email at info@urncst.com | Facebook, Twitter and LinkedIn: @ URNCST

Submit YOUR manuscript today at https://www.urncst.com! 